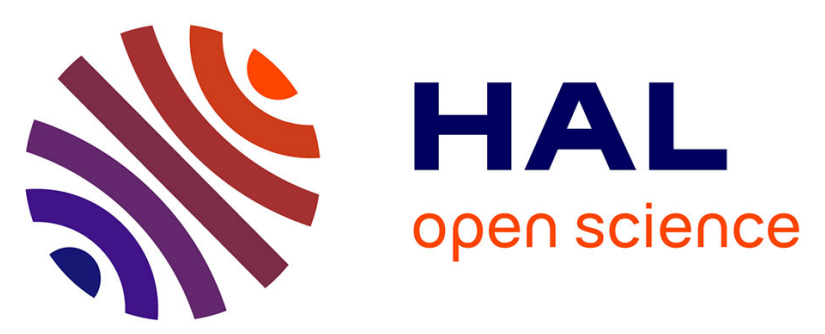

\title{
Unsupervised Creation of Normalization Dictionaries for Micro-Blogs in Arabic, French and English
}

\author{
Amal Htait, Sébastien Fournier, Patrice Bellot
}

\section{To cite this version:}

Amal Htait, Sébastien Fournier, Patrice Bellot. Unsupervised Creation of Normalization Dictionaries for Micro-Blogs in Arabic, French and English. Computación y sistemas, 2018, 19th International Conference on Computational Linguistics and Intelligent Text Processing (CICLing 2018), 22 (3), pp.729-737. 10.13053/CyS-22-3-3034 . hal-01958675

\section{HAL Id: hal-01958675 \\ https://hal.science/hal-01958675}

Submitted on 13 Jun 2019

HAL is a multi-disciplinary open access archive for the deposit and dissemination of scientific research documents, whether they are published or not. The documents may come from teaching and research institutions in France or abroad, or from public or private research centers.
L'archive ouverte pluridisciplinaire HAL, est destinée au dépôt et à la diffusion de documents scientifiques de niveau recherche, publiés ou non, émanant des établissements d'enseignement et de recherche français ou étrangers, des laboratoires publics ou privés. 


\title{
Unsupervised Creation of Normalization Dictionaries for Micro-Blogs in Arabic, French and English
}

\author{
Amal Htait ${ }^{1,2}$, Sébastien Fournier ${ }^{1,2}$, Patrice Bellot ${ }^{1,2}$ \\ ${ }^{1}$ Aix Marseille Univ, Université de Toulon, CNRS, LIS, Marseille, France \\ ${ }^{2}$ Aix Marseille Univ, Avignon Université, CNRS, EHESS, OpenEdition Center, Marseille, France \\ firstname.lastname@lis-lab.fr, firstname.lastname@openedition.org
}

\begin{abstract}
Text normalization is a necessity to correct and make more sense of the micro-blogs messages, for information retrieval purposes. Unfortunately, tools and resources of text normalization are rarely shared. In this paper, an approach is presented based on an unsupervised method for text normalization using distributed representations of words, known also as "word embedding", applied on Arabic, French and English Languages. In addition, a tool will be supplied to create dictionaries for micro-blogs normalization, in a form of pairs of misspelled word with its standard-form word, in the languages: Arabic, French and English. The tool will be available as open source ${ }^{1}$ including the resources: word embedding's models (with vocabulary size of 9 million words for Arabic language model, 5 million words for English language model and 683 thousand words for French language model), and also three normalization dictionaries of 10 thousand pairs in Arabic language, 3 thousand pairs in French language and 18 thousand pairs in English language. The evaluation of the tool shows an average in Normalization success of $96 \%$ for English language, $89.5 \%$ for Arabic Language and $85 \%$ for French Language. Also, the results of using an English language normalization dictionary with a sentiment analysis tool for micro-blog's messages, show an increase in f-measure from 58.15 to 59.56 .
\end{abstract}

Keywords. Normalization, dictionaries, word embedding, micro-blogs, unsupervised, multilingual, Arabic, French.

\footnotetext{
${ }^{1}$ https://github.com/amalhtait/NormAFE https://github.com/OpenEdition/NormAFE http://amalhtait.com/tools.html
}

\section{Introduction}

Twitter and other micro-blogging services are considered as a source of large-volume real-time data, which make them highly attractive for information extraction and text mining. Unfortunately, the quality of micro-blogs' text, with the typos, misspellings, phonetic substitutions and ad hoc abbreviations creates huge obstacles in the way of text processing. Therefore, normalization techniques are a necessity to correct and make more sense of the micro-blogs messages.

This work is inspired by Sridhar et al. [1], an unsupervised method for text normalization using distributed representations of words, known also as "word embedding". The method was not applied on Arabic language, nor French. Also the resources of the previous work were never publicly shared. Therefore, in addition to this work, a tool will be supplied to create dictionaries for micro-blogs normalization, in a form of pairs of misspelled word with its standard-form word, in the languages: Arabic, French and English.

The tool will be available as open source ${ }^{1}$ including: three word embedding's models, with vocabulary size of 9 million words for Arabic language model, 5 million words for English language model and 683 thousand words for French language model. And three normalization dictionaries of 10 thousand pairs in Arabic language, 3 thousand pairs in French language and 18 thousand pairs in English language.

This paper is presented as below: 
- The word embedding's models training: strategy, parameters and datasets.

- The exemplary list of standard-form words (spelled correctly) used as seeds for the creation of the dictionaries.

- The method applied using the models and the seeds list (with their antonyms), to extract for each seed its list of misspelled words followed by a post-processing, or filtering, for more accurate results.

- The evaluation of the tool and dictionaries.

\section{Related Work}

The primary approach in text normalization was the noisy channel model [2], the approach aims to find $\operatorname{argmax} P(S \mid T)$ where the misspelled text is $T$ and its corresponding standard form is $S$, and that's by computing $\operatorname{argmax} P(T \mid S) P(S)$, in which $P(S)$ is a language model and $P(T \mid S)$ is an error model. For many applications, there was a considerable energy to improve both models, with a result of improvement in overall system accuracy. For example, some researchers worked on a new error model for spelling correction, based on generic string to string edits [3]. And others expanded the error model by analyzing a sample of texting forms to define frequent word formation processes in creative texting language [3]. The noisy channel model in text normalization showed effectiveness, but its methods are based on the assumption that a token $t_{i} \in T$ only depends on $s_{i} \in S$, ignoring the context around the token, which can cause ambiguity between words (e.g. gooood was meant to be good or God?).

Statistical machine translation (SMT), has been also used as a method for text normalization, by treating the misspelled text as the source language, and the standard form as the target language. Similar work is found on phrase-based SMT model for text normalization with bootstrapping the phrase alignment [5]. Unfortunately, SMT approaches tend to face a lack of training data.

Some researchers used speech recognition to solve text normalization issue [6].
They converted the input text tokens into phonetic tokens, then restored them to words using phonetic dictionary. Others used a classifier to detect misspelled words, and generates correction possibilities based on morphophonemic similarity [7]. But these methods need large-scale of annotated training data, which limits their adaptability to new domains and languages.

To overcome the limitations of previously cited methods, a technique is applied to learn distributed representation of words (also called word embedding or continuous space representation of words), and to capture distributional similarity between words in a unsupervised manner. As a result, each word will be represented by a numeric vector of high-dimensionality, encoding many linguistic regularities and patterns, also syntactic and semantic word relationships. Due to this representation, words with semantic similarity are represented by similar vectors. Therefore, a misspelled word is represented by a similar vector as its standard-form word.

Sridhar et al. [1] were first to propose that method with a training dataset of 27356 English SMS phrases. His research was the base of several similar work in Portuguese [8], Turkish [9] and Chinese [10], but never in Arabic nor French. In addition, none of these work is open source, and they didn't share the word embedding models, nor the lexicons or dictionaries. Also, all their work was based on relatively small datasets.

For example, Bertaglia's work [8] was focused on products reviews, that are slightly effected by the misspelling errors, the slang words and the typo errors, compared to the tweets, which leads to a much more effective work in micro-blogs' normalization. Also Bertaglia's work [8] was based on a dataset of only 86 thousand products reviews and an unknown small amount of tweets in Portuguese.

And like the rest of the previously cited researchers, the datasets and word embedding models were not publicly shared. This paper focuses on tweets as a dataset resource, with their richness in misspellings and slang words. As a language, it is not limited with one language, but it presents the work with three languages: Arabic, French and English. And as a dataset size, a 
large corpora of tweets is used to create the word embedding models: one billion tweets in English language, 238 million tweets in Arabic language and 48 million tweets in French language.

\section{Word Embedding Training}

Word embedding, or distributed representations of words in a vector space, are currently considered to be among a small number of successful applications of unsupervised learning. Also, they are capable of capturing lexical, semantic, syntactic, and contextual similarity between words. In the following subsections, a description of the word embedding's models training dataset, strategy and parameters.

\subsection{Training Dataset}

The word embedding's training datasets was extracted from the archived twitter streams ${ }^{2}$, which is a collection of $\mathrm{JSON}^{3}$ format data from the general twitter stream, available for the purposes of research, history, testing and memory. This collection contains tweets in many languages what allowed the extraction of tweets in the three languages: Arabic , French and English. Randomly, we extracted files of archived twitter streams dated between 2012 and 2017.

A pre-processing is applied on the three corpora, to improve their usefulness:

- The tweets' corpora is tokenized.

- The user names, hyperlinks and emoticons are replaced by uuser, http and sentiment_emoticon.

- Some characters and punctuations were removed.

- And also, the duplicated tweets were eliminated.

As a result one billion tweets in English language were extracted, in addition to 238 million tweets in Arabic language and 48 million tweets in French language.

\footnotetext{
${ }^{2}$ https://archive.org/details/twitterstream

${ }^{3}$ JavaScript Object Notation is an open-standard file format
}

\subsection{Strategy and Parameters}

For the purpose of learning word embedding from the previously prepared corpora (which is raw text), we use Word2Vec [11]. Word2Vec is a widely used method in natural language processing for generating word embedding, and it has two training strategies:

- Continuous Bag-of-Words (CBOW), in which the model is given a sequence of words with a missing one, and it attempts to predict this omitted word.

- Skip-Gram, in which the model is given a word and it attempts to predict its neighboring words.

According to Mikolov et al. [11], Skip-Gram is more efficient in presenting infrequent words than CBOW. And since the purpose is to seek misspelled words, which are relatively infrequent words, therefore, the Skip-gram architecture is chosen to train the models. To train word embedding and create the models, Gensim ${ }^{4}$ framework for Python is used. And for the parameters, the models are trained with word representations of dimensionality 400 , a context window of one and negative sampling for five iterations $(k=5)$.

By applying the previously mentioned strategy to the datasets, three models were created with a vocabulary size of 9 million words for Arabic model, 5 million words for English model and 683 thousand words for French model.

\section{Dictionaries/Resources Creation}

Word embedding models allow to capture the nearest neighbors of a certain word $X$ using the cosine distance between the dimensional vector of that word $X$ and the dimensional vector of each word in the model. The example, in Fig.1, shows that most of the nearest neighbors of the word alors (then in French) are not real French words but the misspellings of the word alors, such as: alrs, allrs, alr, alord, alirs, allors and alorq, in addition to some other words, such as: sachant (knowing in French) and parce (because in French).

\footnotetext{
${ }^{4}$ https://radimrehurek.com/gensim/index.html
} 
And another example, in Arabic language, the word بارد (cold in Arabic) has the nearest neighbors as its misspellings and not real Arabic words, like:

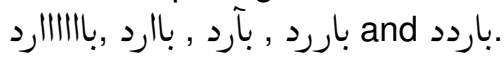

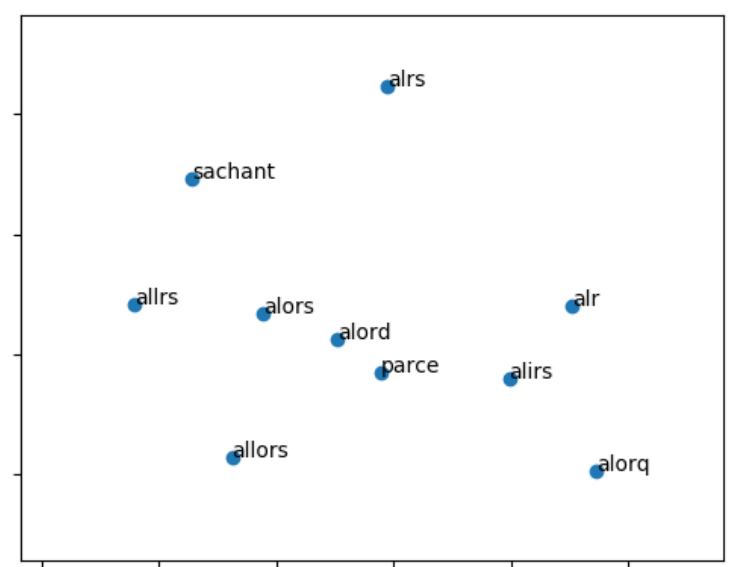

Fig. 1. The French word alors (then) with its nearest neighbors.

Therefore, as a first step in the dictionaries creation, list of standard-form words is needed to capture their misspelled neighbors. In the following subsections, the description of the collected standard-form seed-words lists and the procedure followed for dictionaries creation.

\subsection{Lists of Standard-Form Seed-words}

Most common words in Arabic, French and English were collected for the purpose of creating the standard-form seed-words lists, as below:

- For the Arabic language, the list of 1000 most common Arabic words ${ }^{5}$, the list of 480 Arabic stop-words ${ }^{6}$ and the list of 230 Arabic words highly positive or negative [12] were combined. A list of 1492 Arabic words is the result of the previous lists combination, after removing duplications. The collected list is Standard Arabic words, but dictionaries of any Arabic language dialect can be created (using the

\footnotetext{
${ }^{5}$ http://1000mostcommonwords.com/tag/arabic-words/

${ }^{6} \mathrm{https}: / /$ github.com/stopwords-iso/stopwords$\mathrm{ar} / \mathrm{blob} / \mathrm{master} /$ stopwords-ar.txt
}

shared word embedding Arabic model and tool).

- For the French language, the most common French adjectives and nouns ${ }^{7}$ and the list of 689 French stop-word ${ }^{8}$ were combined. A list of 986 French words is the result of the previous lists combination, after removing duplications.

- For the English language, the 3429 English words from Oxford dictionary ${ }^{9}$ which English stop-words are included, the 500 most frequently used words on twitter ${ }^{10}$ and a list of 90 frequent sentiment words ${ }^{11}$ in tweets [13] were combined. A list of 3501 English words is the result of the previous lists combination, after removing duplications.

To note that the tool and the word embedding models will be publicly available, and more lists of standard-form seed-words (in Arabic, French and English languages) can be added by users to enrich the current dictionaries.

\subsection{Procedure of Dictionaries Creation}

To create a list of possible misspellings (noisy versions) for every standard-form word, two steps are applied:

- First, to determine the similarity between two word embedding, the measure of cosine distance is used between the vectors of standard-form words and the vectors of every other word in the word embedding model. The class most_similar (based on cosine distance measure), of Gensim framework, is used to give a list of 10 most similar words to the standard-form word. To refine the results, most_similar class is used with the antonym of the standard-form word (by Natural Language Toolkit ${ }^{12}$ ) as in the following example:

\footnotetext{
${ }^{7}$ http://www.encyclopedie-incomplete.com/?Les-600-MotsFrancais-Les-Plus

${ }^{8} \mathrm{https}: / / g i t h u b . c o m /$ stopwords-iso/stopwords-fr

${ }^{9} \mathrm{https}: / /$ www.oxfordlearnersdictionaries.com/wordlist/

${ }^{10} \mathrm{http}: / /$ techland.time.com/2009/06/08/the-500-mostfrequently-used-words-on-twitter/

${ }^{11}$ Sentiment words are words highly positive (e.g. Happy) or negative (e.g. Sad)

${ }^{12}$ http://www.nltk.org/
} 
2

model. most_similar ( positive=['active '], negative $=[$ 'inactive '])

The antonyms exclusion eliminates the possibility of extracting the word inactive as a similar word to the word active, since the list of the 5 most similar words of active is: inactive, acitve, avtive, actuve and innactive.

- Second, Python's class SequenceMatcher is applied to compare the previously collected similar words to the standard-form word, for the purpose of eliminating the errors in the list of possible misspellings (e.g., for similar words to the word good $\rightarrow$ goood, goid, great and goos, the word great will be eliminated in this step). The idea of this method is to find the longest contiguous matching subsequence that contains no junk elements (or different elements). The same is then applied recursively to the pieces of the sequences to the left and to the right of the matching subsequence. This method tend to give matches that "look right" to people ${ }^{13}$.

As a result for that procedure, three dictionaries were extracted in the form of pairs of misspelled words with their standard-form word:

- Arabic language dictionary with 10 thousand pairs.

- French language dictionary with 3 thousand pairs.

— English language dictionary with 18 thousand pairs.

\section{Evaluation}

\subsection{Evaluation of Dictionaries' Content}

To evaluate the tool by dictionary's content, a manual annotation is applied by checking the correct pairing between the misspelled words and their assigned standard-form words, and the annotation differentiate between two types of evaluation: Correction (e.g. graet and great) and

\footnotetext{
${ }^{13}$ https://docs.python.org/2/library/difflib.html
}

Normalization which includes the correction and the lemmatization (e.g. shows and show). Table 1 shows an example of the method of annotation, where the check-mark is a right correction or normalization, and the x-mark is a wrong one.

To have as much similar evaluation as possible between the three languages, a sample of 50 standard-form words highly positive or negative is selected, for each language, since sentiment words are usually used in micro-blogs at same frequencies in most languages.

Cosine similarity measure allows to find a list of most similar words of the standard-form word. And the class most_similar of Gensim framework, which is based on cosine similarity measure, has by default 10 as a size of that list. Changing the size of that list can lead to creating larger dictionaries, like in the below example:

- The list of 5 similar words of good is: goood, goid, gooood, goooood, gud.

- The list of 15 similar words of good is: goood, goid, gooood, goooood, gud, gooooood, goos, gpod, great, gopd, giod, gooo00000d, cargood, goooo00000d, g00d.

The second list is larger and richer in words, but it includes unwanted words like great and cargood (since these words are not corrections of the word good). Therefore, and as part of the evaluation, the number of similar words extracted is added as a parameter, and the evaluation is applied by variating its value as $5,25,50$ and 100 .

First, a briefing of the evaluation results are presented below:

- For English language, an average of $96 \%$ in Normalization success, and of $86 \%$ in Correction success.

- For Arabic language, an average of $89.5 \%$ in Normalization success, and of $83.7 \%$ in Correction success.

- For French language, an average of $85 \%$ in Normalization success, and of $73.6 \%$ in Correction success. 
Table 1. An example of dictionaries annotation, where three examples from each language is selected (English, French and Arabic), and where the check-mark is a right correction or normalization, and the x-mark is a wrong one

\begin{tabular}{|c|c|c|c|}
\hline Misspelled & Standard-word & Correction & Normalization \\
\hline gladd & glad & $\checkmark$ & $\checkmark$ \\
\hline hates & hate & $x$ & $\checkmark$ \\
\hline horrific & horrible & $x$ & $x$ \\
\hline aiiiiiiime (loooooove) & aime (love) & $\checkmark$ & $\checkmark$ \\
\hline decevra (will disappoint) & decevoir (disappoint) & $x$ & $\checkmark$ \\
\hline deballer (unpack) & deprimer (depress) & $x$ & $x$ \\
\hline متاذ ( misspelled excellent) & متّاز (excellent) & $\checkmark$ & $\checkmark$ \\
\hline اكرهه (hate him) & اكره (hate) & $x$ & $\checkmark$ \\
\hline اهبل (dump) & غي (stupid) & $x$ & $x$ \\
\hline
\end{tabular}

Table 2. Results of Echo with SemEval2014's data [14], with a baseline of no normalization, then with a normalization applied using four dictionaries that differ in the number of most similar words and in their size

\begin{tabular}{llllll}
\hline Echo & \#SimilarWords & DictSize & LiveJournal2014 & SMS2013 & Twitter2013 \\
\hline baseline & - & - & 58.15 & 55.95 & 55.64 \\
+Dict_1 & 5 & 371 & 58.50 & 55.97 & 55.94 \\
+Dict_2 & 25 & 1449 & 58.67 & 56.16 & 56.16 \\
+Dict_3 & 50 & 2337 & 58.90 & 56.42 & 56.21 \\
+Dict_4 & 100 & 2776 & $\mathbf{5 9 . 5 6}$ & $\mathbf{5 6 . 6 1}$ & $\mathbf{5 6 . 2 2}$ \\
\hline
\end{tabular}

Table 3. An example of Arabic language pairs of dialect word with its standard-form word in the normalization dictionary

\begin{tabular}{lll}
\hline DialectWord & DialectSource & StandardWord \\
\hline غبيط ضي ضيقك (Stupid) \\
ضايقك (bothered you) \\
\hline
\end{tabular}

Then, the results of the evaluation are presented with more details in the graphs of Fig.2, consequentially from top to bottom in English, French and Arabic languages, where the percentage of successful Correction is the line in blue and the percentage of successful Normalization is the line in red, both calculated relatively to the variation of most similar words number (as 5, 25, 50 and $100)$ and the variation of the dictionaries' size (the bars in grey). To note that the created dictionaries for the Arabic language reached the size of 2053 pairs when selecting 100 most similar words, for the
French language the size of 500 pairs, and for the English language the size of 2776 pairs.

The results in Fig.2 shows that the percentage of successful Normalization is always higher than the percentage of successful Correction. Also, for English (upper graph) and French (middle graph) languages, an increase in the percentage of successful Correction and Normalization appears when the number of similar words extracted is between 5 and 25, followed by a continuous decrease. And for Arabic language (lower graph), a sharp decrease with the percentage of successful Correction and Normalization is observed, with the increase of similar words extracted number.

\subsection{English Dictionary Evaluation with Sentiment Analysis Tool}

The evaluation of the dictionaries by their content, in the previous section, shows promising results. But to prove the usefulness of the dictionaries, the effect of the English language normalization dictionary is 


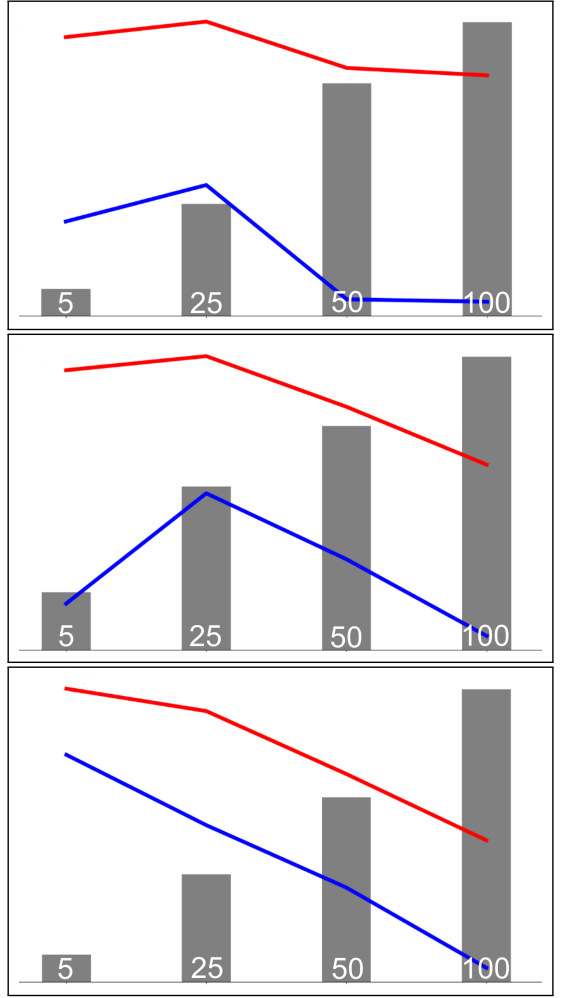

Fig. 2. The percentage of successful Correction (blue) and Normalization (red) in the test dictionaries (dictionaries created based on 50 sentiment standard-words), both calculated relatively to the variation of most similar words number (as 5,25 , 50 and 100) and the variation of the dictionaries' size (the bars in grey), consequentially from top to bottom, in English, French and Arabic languages

evaluated by predicting sentiment polarity (positive, negative or neutral) in micro-bogs' messages.

Echo ${ }^{14}$, an open source software for sentiment analysis based on supervised machine learning algorithm, is used as a test tool. And as training and testing datasets, SemEval2014's datasets [14] are used: for training, the annotated (by sentiment polarity) training dataset of almost 10000 Tweets, and for testing the 1000 Live-Journal from 2014, the 2000 SMS from 2013 and the 3800 Tweets from 2013.

\footnotetext{
${ }^{14}$ https://github.com/OpenEdition/echo
}

The results ${ }^{15}$ of Echo, predicting sentiment polarity of the testing data, are presented in Table 2.

The first row is the baseline, where Echo runs without normalization. Then, for the rest of the rows, the normalization was applied using four dictionaries, all based on the same list of 50 English sentiment standard-words, but differ in the number of most similar words chosen at the level of dictionary creation $(5,25,50$ or 100$)$, and as a result, these dictionaries differ in their size (since the size of a dictionary increases with the increasing number of "most similar words").

The results, in Table 2, show an increase in the capability of Echo to successfully predict sentiment polarity in micro-blogs's messages, using the normalization dictionaries. Also, they show that Echo achieves better results when increasing the dictionary size, and best results in this evaluation is achieved with dictionary size equals to 2776 pairs.

\section{Conclusion}

This paper presents an approach based on an unsupervised method for text normalization using word embedding, applied on Arabic, French and English languages. In addition, a tool will be supplied to create dictionaries for micro-blogs normalization, and will be available as open source including the resources: three word embedding models, and three normalization dictionaries, for the three languages: Arabic, French and English. The evaluation of the tool shows an average in Normalization success of $96 \%$ for English language, $89.5 \%$ for Arabic Language and $85 \%$ for French language. Also the results of using an English language normalization dictionary on a sentiment analysis tool for micro-blog's messages, show an increase in the tool's ability to predict the sentiment polarity of the messages.

The evaluations' results in Sect. 5.1 show that while the dictionary's size increases, the percentage of Normalization and Correction success decreases. But, on the other hand, and based on the evaluation in Sect. 5.2,

\footnotetext{
${ }^{15}$ The results are displayed with the f-measure value, a measure of a test's accuracy [15].
} 
the effectiveness of the dictionary (in sentiment analysis) increases with its size, independently from the percentage of success in Normalization and Correction.

Finally, this work can be a resource for many domains in Natural Language Processing. For example, by observing the Arabic language dictionary, many pairs of dialect word with its standard-form word were found, some examples are in Table 3. Also, in the creation process of the word embedding models, a large number of emoticons and emojis (expressing sentiment, like $\odot$ ) were replaced by expressions: positive_emoticon, negative_emoticon and neutral_emoticon, for the purpose of a future use of these models in sentiment analysis tasks.

\section{Acknowledgments}

This work has been supported by the French State, managed by the National Research Agency under the «Investissements d'avenir» program under the EquipEx DILOH projects (ANR-11-EQPX-0013).

\section{References}

1. Sridhar, V. K. R. (2015). Unsupervised text normalization using distributed representations of words and phrases. Proceedings of the 1st Workshop on Vector Space Modeling for Natural Language Processing, pp. 8-16.

2. Shannon, C. E. (1948). A Mathematical Theory of Communication. The Bell System Technical Journal, Vol. 27, No. 3, pp. 379-423.

3. Brill, E. \& Moore, R. C. (2000). An improved error model for noisy channel spelling correction. Proceedings of the 38th Annual Meeting on Association for Computational Linguistics, pp. 286-293.

4. Cook, P. \& Stevenson, S. (2009). An unsupervised model for text message normalization. Proceedings of the workshop on computational approaches to linguistic creativity, Association for Computational Linguistics, pp. 71-78.
5. Aw, A., Zhang, M., Xiao, J., \& et al. (2006). A phrase-based statistical model for SMS text normalization. Proceedings of the COLING/ACL on Main conference poster sessions, Association for Computational Linguistics, pp. 33-40.

6. Kobus, C., Yvon, F., \& Damnati, G. (2008). Transcrire les SMS comme on reconnaît la parole. Actes de la Conférence sur le Traitement Automatique des Langues (TALN'08), pp. 128-138.

7. Han, B. \& Baldwin, T. (2011). Lexical normalisation of short text messages: Makn sens a\# twitter. Proceedings of the 49th Annual Meeting of the Association for Computational Linguistics: Human Language TechnologiesVolume 1, Association for Computational Linguistics, pp. 368-378.

8. Bertaglia, T. F. C. \& Nunes, M. d. G. V. (2016). Exploring Word Embeddings for Unsupervised Textual User-Generated Content. Normalization. Proc. 26th Int'l Conf. Computational Linguistics (COLING 16), pp. 112-120.

9. Eryiğit, G. \& Torunoğlu-Selamet, D. (2017). Social media text normalization for Turkish. Natural Language Engineering, pp. 1-41.

10. Yan, X., Li, Y., \& Fan, W. (2017). Identifying domain relevant user generated content through noise reduction: a test in a Chinese stock discussion forum. Information Discovery and Delivery, Vol. 45, No. 4, pp.181-193.

11. Mikolov, T., Chen, K., Corrado, G., \& et al. (2013). Efficient estimation of word representations in vector space. ICLR Workshop Papers.

12. Salameh, M., Mohammad, S., \& Kiritchenko, S. (2015). Sentiment after Translation: A Case-Study on Arabic Social Media Posts. Proceedings of the conference the North American chapter of the association for computational linguistics: Human language technologies, pp. 767-777. 
13. Htait, A., Fournier,S., \& Bellot, P. (2017). LSIS at SemEval Task 4: Using Adapted Sentiment Similarity Seed Words For English and Arabic Tweet Polarity Classification. Proceedings of the 11th International Workshop on Semantic Evaluation (SemEval-2017), pp. 718-722.

14. Rosenthal, S., Nakov, P., Ritter, A., \& Stoyanov, V. (2014). SemEval-2014 Task 9: Sentiment analysis in Twitter. Proceedings of the 8th International Workshop on Semantic the 8th International Workshop on Semantic Evaluation (SemEval 14), pp. 73-80.

15. Powers, D. M. (2011). Evaluation: from precision, recall and F-measure to $\mathrm{ROC}$, informedness, markedness and correlation. Journal of Machine Learning Technologies, 2(1), pp. 37-63.

Article received on 20/01/2018; accepted on 05/03/2018. Corresponding author is Amal Htait. 